\title{
SOME RESULTS ON BOX SPLINES
}

\author{
BY WOLFGANG DAHMEN AND CHARLES A. MICCHELLI
}

\begin{abstract}
The purpose of this note is to describe recent progress made in understanding the box spline.
\end{abstract}

Introduction. The purpose of this note is to describe progress made in understanding the box spline. This function is one of several polyhedral splines intensively studied during the past few years; see [1] for a survey of this subject.

For any set $X=\left\{x^{1}, \ldots, x^{n}\right\} \subseteq R^{s} \backslash\{0\}$ with $\langle X\rangle:=$ linear span of $X=R^{s}$, the box spline is defined by requiring that

$$
\int_{R^{\circ}} B(x \mid X) f(x) d x=\int_{0}^{1} \cdots \int_{0}^{1} f\left(\sum_{i=1}^{n} t_{i} x^{i}\right) d t_{1} \cdots d t_{n}
$$

holds for all continuous functions on $R^{s} . \quad B(x \mid X)$ is a smooth piecewise polynomial of degree $n-s$ and continuity class $C^{d(X)-1}\left(R^{s}\right)$, where

$$
d(X)=\max \left\{m:\langle X \backslash Y\rangle=R^{s}, \forall Y \subset X \ni|Y|=m\right\} .
$$

We are interested in the spline space spanned by integer translates of the box spline

$$
S(X)=\left\langle\left\{B(0-\alpha \mid X): \alpha \in Z^{s}\right\}\right\rangle \text {. }
$$

It is important to know for the purpose of approximating smooth functions by scaled translates of box splines what polynomials are in $S(X)$. Denoting by $\Pi\left(R^{s}\right)$ the set of all polynomials on $R^{s}$, and by $D^{\prime}\left(R^{s}\right)$ the space of Schwartz distributions on $C_{0}^{\infty}\left(R^{s}\right)$, it is known that for $X \subset Z^{s}$,

$$
S(X) \cap \Pi\left(R^{s}\right)=D(X)
$$

where

$$
D(X)=\left\{f \in D^{\prime}\left(R^{s}\right): D_{Y} f=0, \forall Y \subset X \ni\langle X \backslash Y\rangle \neq R^{s}\right\}
$$

and $D_{Y}=\prod_{y \in Y} D_{y}, D_{y}$ being the directional derivative in the direction of $y$.

The Nullstellensatz can be used to show that $\operatorname{dim} D(X)<\infty$ and $D(X) \subset$ $\Pi\left(R^{s}\right)$. This fact and the others mentioned above, as well as relevant references, appear in [1].

Theorems. Our first result is

Theorem 1. For any $X \subset R^{s} \backslash\{0\}$ with $\langle X\rangle=R^{s},|X|<\infty$, one has

$$
\operatorname{dim} D(X)=|B(X)|
$$

where $B(X)=\left\{Y: Y \subset X,|Y|=s,\langle Y\rangle=R^{s}\right\}$.

Received by the editors August 10, 1983 and, in revised form, December 6, 1983. 1980 Mathematics Subject Classification. Primary 41A63, 41A15, 47B39, 10E05.

(C) 1984 American Mathematical Society $0273-0979 / 84 \$ 1.00+\$ .25$ per page 
Several special cases of this result can be proved directly, for instance when the vectors in $X$ are in general position or are just multiples of the coordinate vectors. The general case is harder. We employ an induction on $s$ and $|X|=n$ to construct a basis for $D(X)$.

For the next theorem we introduce

$$
B(X)=\left\{x=\sum_{i=1}^{n} t_{i} x^{i}: 0 \leq t_{i} \leq 1\right\}
$$

and note that $B(X)=\operatorname{supp} B($ 이X).

THEOREM 2.

$$
\operatorname{vol}_{s} B(X)=\sum_{Y \in B(X)}|\operatorname{det} Y|
$$

Sketch of Proof. For every $Y \in B(X)$, the set $B(Y)$ is the parallelepiped spanned by $Y$. One can show that $B(X)$ can be covered by a union of translates of such sets with disjoint interiors. From this fact Theorem 2 is immediate.

In the remainder of our discussion we require that $X \subset Z^{s}$. The main application of Theorems 1 and 2 is to completely settle the question of local linear independence of translates of box splines. To this end, we will always use $\Omega$ for any region on which all the translates of a box spline are polynomials. The local span of box splines relative to $\Omega$ is defined by

$$
S(X \mid \Omega)=\left\langle\left\{B(x-\alpha \mid X): \forall \alpha \in Z^{s} \ni \operatorname{supp} B(\circ-\alpha \mid X) \cap \Omega \neq \varnothing\right\}\right\rangle .
$$

First we count the number of translates whose support intersects $\Omega$ (thereby obtaining an upper bound on $\operatorname{dim} S(X \mid \Omega)$ ).

Theorem 3. Let $X \subset Z^{s},\langle X\rangle=R^{s}$ and define

$$
b(\Omega \mid X)=\left\{\alpha: \alpha \in Z^{s} \ni \operatorname{supp} B(\circ-\alpha \mid X) \cap \Omega \neq \varnothing\right\} ;
$$

then $|b(\Omega \mid X)|=\operatorname{vol}_{s} B(X)$.

The proof of this result depends on the decomposition of supp $B($ 이 $X)$ into translates of the parallelepipeds $B(Y), Y \in B(X)$, used in Theorem 2, and the observation that any $\Omega$ is covered by the same number of translates of parallelepipeds $B(Y)$.

THEOREM 4. (Local linear independence of box splines). Let $X \subset Z^{s},\langle X\rangle=$ $R^{s}$. Then $S(X \mid \Omega)=D(X)$. Furthermore, the following three conditions are equivalent.

(i) $\sum_{\alpha} c_{\alpha} B(x-\alpha \mid X)=0, x \in \Omega$, implies $c_{\alpha}=0, \alpha \in b(\Omega \mid X)$.

(ii) For any $Y \in B(X)$, we have $|\operatorname{det} Y|=1$.

(iii) $\sum_{\alpha} c_{\alpha} B(x-\alpha \mid X)=0, x \in R^{s}$, implies $c_{\alpha}=0, \alpha \in \mathbf{Z}^{s}$.

Sketch of Proof. The first assertion follows from the fact that the map $(T f)(x)=\sum_{\alpha} f(\alpha) B(x-\alpha \mid X)$ is one-to-one and onto $D(X)$, and the observation that $\left.B(0-\alpha \mid X)\right|_{\Omega} \in D(X)$ (see [1]). The equivalence between (ii) and (iii) is known [1], while the local linear independence (i) follows from Theorems 1, 2 and 3. 
In general, to characterize the linear dependence relations among translates of box splines we need the difference operator analog of $D(X)$ given by

$$
\Delta(X)=\left\{f: Z^{s} \rightarrow \mathbf{C}: \Delta_{Y} f=0, \forall Y \subset X, \ni\langle X \backslash Y\rangle \neq R^{s}\right\}
$$

where

$$
\left(\Delta_{Y} f\right)(\alpha)=\left(\prod_{y \in Y} \Delta y\right) f(\alpha), \quad(\Delta y f)(\alpha)=f(\alpha+y)-f(\alpha) .
$$

First we give a complete characterization of $\Delta(X)$.

Theorem 5. If $A(X)=\left\{z \in \mathbf{C}^{s}: \exists Y \in B(X), \ni z^{y}=1, y \in Y\right\}$ and $X_{z}=$ $\left\{y: y \in X, z^{y}=1\right\}$ then $f \in \Delta(X)$ if and only if

$$
f(\alpha)=\sum_{z \in A(X)} z^{\alpha} p(\alpha \mid z),
$$

where $p(x \mid z) \in D\left(X_{z}\right)$ and, moreover,

$$
\operatorname{dim} \Delta(X)=\operatorname{vol}_{s} B(X) \text {. }
$$

Proof. Induction on $|X|$ yields $\operatorname{dim} \Delta(X) \leq \operatorname{vol}_{s} B(X)$. On the other hand, one can show that the above sequences are in $\Delta(X)$. Theorems 1 and 2 can then be used to count $\operatorname{vol}_{s} B(X)$ linearly independent such sequences.

REMARK. Since $(1, \ldots, 1) \in A(X)$ and $D(X)$ only contains polynomials, we see by Theorem 5 that $f \in \Delta(X)$ if and only if $f=f_{1}+f_{2}$, where $f_{1} \in D(X)$ and $f_{2}$ satisfies

$$
f_{2}(\alpha)=\sum_{z \in A(X) \backslash\{(1, \ldots, 1)\}} z^{\alpha} p(\alpha \mid z),
$$

where $p(x \mid z) \in D\left(X_{z}\right)$.

Let $E(X)$ denote the subspace of $\Delta(X)$ consisting of all elements of the form (1). Combining the above result with Poisson's summation formula yields

THEOREM 6. If $f \in E(X)$ then

$$
\sum_{\alpha} f(\alpha) B(x-\alpha \mid X)=0, \quad x \in R^{s} .
$$

Next we solve the initial value problem for the family of difference operators $\Delta_{Y},\langle X \backslash Y\rangle \neq R^{s}$, which determine $\Delta(X)$.

TheOREM 7. Any sequence $\left\{d_{\alpha}: \alpha \in b(\Omega \mid X)\right\}$ has a unique extension $\left\{c_{\alpha}: \alpha \in Z^{s}\right\}$ in $\Delta(X)$.

Sketch of Proof. From Theorems 3 and 5 we known that $\operatorname{dim} \Delta(X)=$ $|b(\Omega \mid X)|$. One can then use induction on $|X|$ to show that any extension of $d_{\alpha}=0, \alpha \in b(\Omega \mid X)$, in $\Delta(X)$ must vanish identically.

Corollary 1. Suppose, for any $Y \in B(X),|\operatorname{det} Y|=1$. Then for any data $y_{\alpha}, \alpha \in b(\Omega \mid X)$, there is a unique $p \in D(X)$ such that $p(\alpha)=y_{\alpha}, \alpha \in b(\Omega \mid X)$.

This result is used to construct a linear projector onto $S(X)$. The construction of this map and details of the proofs will be given elsewhere. 


\section{REFERENCES}

1. W. Dahmen and C. A. Micchelli, Recent progress on multivariate splines, Approximation Theory IV (C. K. Chui, L. L. Schumaker and J. D. Ward, eds.), Academic Press, New York, 1983, pp. 27-121.

Fakultät für Mathematik, Universität Bielefeld, Universitätsstrasse, 48 Bielefeld, West Germany

IBM Thomas J. Watson Research Center, P. O. Box 218, Yorktown Heights, NEW YORK 10598 